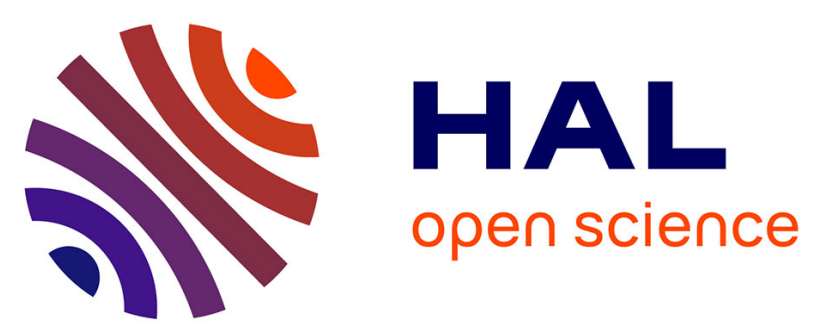

\title{
3D Head-Mounted Antenna Array Architecture Optimization based on the Fisher Information Matrix
}

D Poirier-Quinot, P Duvaut, L Girardeau, Brian F,g Katz

\section{To cite this version:}

D Poirier-Quinot, P Duvaut, L Girardeau, Brian F,g Katz. 3D Head-Mounted Antenna Array Architecture Optimization based on the Fisher Information Matrix. Ultra Modern Telecommunications and Control Systems and Workshops (ICUMT), 2012 4th International Congress on, Oct 2012, St. Petersburg, Russia. pp.135 - 142, 10.1109/ICUMT.2012.6459653 . hal-01108665

\section{HAL Id: hal-01108665 \\ https://hal.science/hal-01108665}

Submitted on 23 Jan 2015

HAL is a multi-disciplinary open access archive for the deposit and dissemination of scientific research documents, whether they are published or not. The documents may come from teaching and research institutions in France or abroad, or from public or private research centers.
L'archive ouverte pluridisciplinaire HAL, est destinée au dépôt et à la diffusion de documents scientifiques de niveau recherche, publiés ou non, émanant des établissements d'enseignement et de recherche français ou étrangers, des laboratoires publics ou privés.

\section{(ㄷ)(1) $\$$}

Distributed under a Creative Commons Attribution - NonCommerciall 4.0 International 


\title{
3D Head-Mounted Antenna Array Architecture Optimization based on the Fisher Information Matrix
}

\author{
D. Poirier-Quinot ${ }^{\dagger \pi \varepsilon}$, P. Duvaut ${ }^{\varepsilon}$, L. Girardeau ${ }^{\dagger}$, B.F.G. Katz ${ }^{\pi}$ \\ ${ }^{\dagger}$ EADS Astrium Services, 92150 Suresnes, France \\ ${ }^{\varepsilon}$ ETIS / ENSEA - Université Cergy-Pontoise - CNRS, 95000 France \\ ${ }^{\pi}$ LIMSI-CNRS, Université Paris Sud, 91403 Orsay, France
}

\begin{abstract}
This paper introduces a method to optimize the configuration of a 3D helmet-mounted antenna array carried by emergency rescuers expected to locate natural disaster survivors. Configuration optimization is based on metrics extracted from the 3D Fisher Information Matrix (FIM) relative to the considered array architecture for single source Direction Of Arrival (DOA) estimation. Comprehensive simulations illustrate the optimization metrics behaviour with regard to survivor's emitter 3D DOA and array configuration. Design constraints related to helmetmounted antenna requirements reduce the usually exhausting FIM based design optimization to a 2 degrees of freedom exploration of the array DOA estimation performances.

Index Terms-Direction Of Arrival Estimation, Fisher Information Matrix, Helmet-mounted Antenna Array
\end{abstract}

\section{INTRODUCTION}

To efficiently rescue survivors in the aftermath of earthquakes or tsunamis, it is of paramount importance to know where to aim the research once the emergency team is deployed on the field. In these situations, one may reasonably assume that most survivors carry a mobile phone, even one without GPS capability in the worst-case scenario. In such conditions, the rescue protocol requires a local activation of these mobile phones to allow Direction Of Arrival (DOA) estimation process. These estimations are based on the electromagnetic measurements collected through the antenna array carried by the rescuers. To individually reach and assist as many survivors as possible, rescuers would need to be equipped with handfree antenna arrays that could estimate their DOAs on the go [1].

While very well known high resolution DOA estimation methods (MUSIC [2], ESPRIT [3], WSF [4], etc.) exist in the literature along with extensive studies concerning 2D and 3D antenna array optimization ([5],[6]), design optimization of helmet-mounted devices remains a challenge. The involved dimensions and geometry constraints obviously raise several issues compared to usually considered arrays. This paper is part of a broad project that assumes a network operator substitution on the local wireless network, which ensures the ability to activate one handset at a time therefore reducing the considered scenario to a single source 3D DOA estimation in a noised environment. A few papers tackle the 3D antenna array optimization problem based on the Fisher Information Matrix (FIM) in the single source case ([7],[8]), but for general array configurations not adapted to hereafter considered architecture. This paper thus introduces a dedicated optimization method for 3D helmet-mounted antenna arrays based on metrics derived from the 3D FIM.

The remainder of the paper is organized as follows. Section 2 presents the geometrical configuration of the helmetmounted antenna array and introduces the considered signal model. Section 3 details the geometrically oriented form of the 3D FIM for the considered model while its derivation is given in Appendix A. Section 4 suggests antenna array architecture optimization metrics extracted from the 3D FIM. Finally, Section 5 presents simulation results and analysis on suggested metrics with relation to DOA angle and array configuration while Section 6 holds the conclusion of this paper.

\begin{tabular}{|cl|}
\hline Notations & \\
$\mathrm{E}(\cdot)$ & statistical expectation \\
$\Re\{\cdot\}$ & real part of a complex element \\
$(\cdot)^{\dagger}$ & conjugate transpose \\
$|\cdot|$ & determinant of a matrix \\
$\operatorname{tr}(\cdot)$ & trace operator \\
$\langle\cdot . \cdot\rangle$ & scalar product \\
$I_{N}$ & the $\mathrm{N} \times \mathrm{N}$ identity matrix \\
$T$ & sample length \\
$N$ & number of array sensors \\
$\operatorname{col}\left[u_{n}\right]$ & column vector of elements $u_{n}, n \in \llbracket 1, N \rrbracket$ \\
$\operatorname{diag}\left(u_{n}\right)$ & diagonal matrix of elements $u_{n}, n \in \llbracket 1, N \rrbracket$ \\
$\delta_{\xi}(\cdot)$ & $1^{s t}$ partial derivative wrt $\xi$ \\
$\delta_{\xi, \zeta}^{2}(\cdot)$ & $2^{\text {nd }}$ order partial derivative $w r t \xi$ and $\zeta$ \\
\hline & \\
\hline
\end{tabular}

\section{Antenna Array Configuration \& Signal Model}

We consider the 3 elements spherical array depicted in Figure 1. Each supposedly isotropic and identical sensor $M_{i}$ for $\mathrm{i} \in \llbracket 1,3 \rrbracket$ evolves on a sphere of radius $r$ centered at $O$. $\mathbf{O M}_{1}$ and $\mathbf{O M}_{3}$ (resp. $\mathbf{O M}_{2}$ ) belongs to the cartesian plane 
$O x y$ (resp. $O z x$ ), their angular positions being defined by angles $\pm \beta$ (resp. $\eta$ ). In keeping with Figure 1 and according to the activation scenario, the single source $S$ propagates in free space at radio wavelength $\lambda$ from angle coordinates $(\theta, \varphi)$ while the two angular degrees of freedom of the helmetmounted antenna array are $(\beta, \eta)$. For notational convenience, $(\theta, \varphi)$ and $(\beta, \eta)$ will also be denoted as $\Theta$ and $\Omega$, or even omitted in thereafter derivations whenever there is no possible confusions.

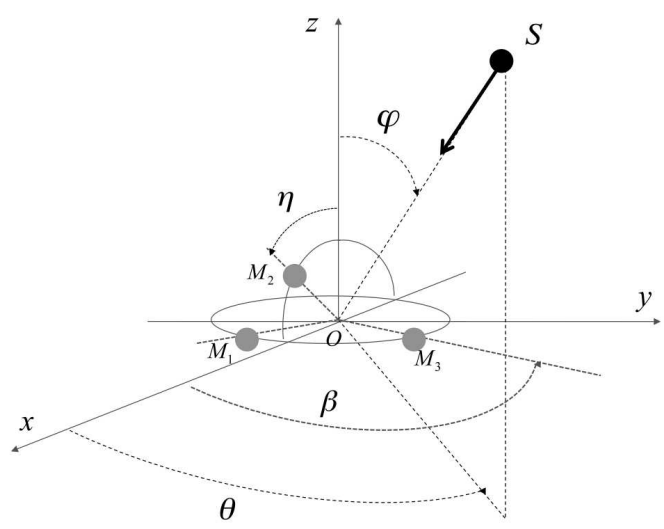

Fig. 1. Helmet-mounted antenna for single source $S$ location

Under single wavelength propagation conditions, in the absence of noise, the complex amplitude vector $\mathbf{e}_{\boldsymbol{\Omega}}$ of the received signal on the array sensors takes the following form

$$
\mathbf{e}_{\boldsymbol{\Omega}}(\boldsymbol{\Theta})=\left(\begin{array}{c}
e^{j \frac{2 \pi r}{\lambda}(\sin (\varphi) \cos (\theta+\beta))} \\
e^{j \frac{2 \pi r}{\lambda}(\sin (\varphi) \cos (\theta) \sin (\eta)+\cos (\varphi) \cos (\eta))} \\
e^{j \frac{2 \pi r}{\lambda}(\sin (\varphi) \cos (\theta-\beta))}
\end{array}\right)
$$

Consequently, the vector measurement used to perform the DOA estimation is

$$
\mathbf{y}[t]=\mathbf{e}_{\boldsymbol{\Omega}}(\boldsymbol{\Theta}) s[t]+\mathbf{n}[t]
$$

where $s[t] \in \mathbb{C}$ is one of the learning sequences of the considered wireless protocol, at this stage assumed to be a stochastic zero mean white Gaussian noise, while $\mathbf{n}[t]$ $\in \mathbb{C}^{M \times 1}$ is supposed to be a complex, circular, temporally and spatially white Gaussian noise. Respective signal and noise variances are denoted $\sigma_{s}^{2}$ and $\sigma_{n}^{2}$.

The next section is focused on the associated Fisher Information Matrix, subsequently used in the array configuration optimization. A complete study on the 3D FIM derivation can be found in [9] for general array architectures, not necessarily formulated to apprehend the simple optimization method adopted in this paper.

\section{FISHER INFORMATION MATRIX AND ASSOCIATED STOCHASTIC CRAMER-RAO BOUND}

As a reminder, the Cramer-Rao Bound (CRB) is a lower bound of the covariance matrix of any unbiased estimator, first introduced by R.A. Fisher in [10], defined as the inverse of the Fisher Information Matrix. Various expressions and applications of the CRB may be found in the literature, e.g. for MUSIC (MUltiple SIgnal Classification) performances analysis in [2] or [11].

Defining the Fisher Information Matrix as $F(\Theta)=$ $-E\left[\frac{\partial^{2} \Lambda(\mathbf{\Theta})}{\partial \mathbf{\Theta}^{2}}\right]$, the Cramer-Rao theorem states that

$$
\Gamma(\hat{\boldsymbol{\Theta}})-F(\boldsymbol{\Theta})^{-1}
$$

is a positive definite matrix, where $\Gamma(\hat{\boldsymbol{\Theta}})$ is the covariance matrix of $\hat{\boldsymbol{\Theta}}$, unbiased estimator of $\boldsymbol{\Theta}$. Due to the exponential nature of the steering vector elements in $\mathbf{e}_{\boldsymbol{\Omega}}(\boldsymbol{\Theta})=$ $\left[e^{j \frac{2 \pi r}{\lambda} u_{n}(\boldsymbol{\Omega}, \boldsymbol{\Theta})}\right]$, the aforementioned derivative leads to define the steering derivative vector $\mathbf{v}_{\xi}=\delta_{\xi}(\mathbf{U}) \mathbf{e}_{\boldsymbol{\Omega}}(\boldsymbol{\Theta})$ for $\xi \in$ $\{\theta, \varphi\}$, where $\mathbf{U}=\operatorname{diag}\left(u_{n}(\boldsymbol{\Omega}, \boldsymbol{\Theta})\right)$. After calculations detailed in Appendix A, under the assumptions made in Section II and using the simplified notation e for $\mathbf{e}_{\boldsymbol{\Omega}}(\boldsymbol{\Theta})$, the FIM relevant to our problem takes the following form

$$
F(\boldsymbol{\Theta})=\gamma_{N}\left(\begin{array}{c}
\left\langle\mathbf{v}_{\theta}, \mathbf{v}_{\theta}\right\rangle_{\perp \mathbf{e}}\left\langle\mathbf{v}_{\theta}, \mathbf{v}_{\varphi}\right\rangle_{\perp \mathbf{e}} \\
\left\langle\mathbf{v}_{\varphi}, \mathbf{v}_{\theta}\right\rangle_{\perp \mathbf{e}}\left\langle\mathbf{v}_{\varphi}, \mathbf{v}_{\varphi}\right\rangle_{\perp \mathbf{e}}
\end{array}\right)
$$

with

$$
\gamma_{N}=2 \alpha T \frac{1}{\left(1+\frac{1}{\alpha N}\right)}\left(\frac{2 \pi r}{\lambda}\right)^{2}
$$

where $T$ is the number of measured samples, $\alpha=\sigma_{s}^{2} / \sigma_{n}^{2}$ the linear SNR and $N$ the number of array elements. $\langle., .\rangle_{\perp \mathbf{e}}$ denotes the scalar product weighted by the orthonormal projector on the MUSIC defined noise subspace $\boldsymbol{\Pi}_{\perp \mathbf{e}}=\mathbf{I}_{\mathbf{N}}-\tilde{\mathbf{e}} \tilde{\mathbf{e}}^{\dagger}$, i.e.

$$
\left\langle\mathbf{v}_{\theta}, \mathbf{v}_{\varphi}\right\rangle_{\perp \mathbf{e}}=\mathbf{v}_{\theta}^{\dagger} \boldsymbol{\Pi}_{\perp \mathbf{e}} \mathbf{v}_{\varphi} \quad \text { with } \quad \tilde{\mathbf{e}}=\frac{\mathbf{e}}{\sqrt{\mathbf{e}^{\dagger} \mathbf{e}}}
$$

Note that except the explicit form of $\gamma_{N}$ in (4), equation (3) is valid for any spherical array configuration in the single source DOA estimation scenario.

\section{ARray Optimisation Metrics}

The inversion of (3) being straightforward, let us consider only the upper left and lower right terms of $F(\boldsymbol{\Theta})^{-1}$, lower bounds for the variance of respectively any $\theta$ and $\varphi$ estimation, designated thereafter by $C R B_{\xi, \xi}$ for $\xi \in\{\theta, \varphi\}$. Defining the hereafter two correlation coefficients

$$
\rho^{2}=\frac{\left|\left\langle\mathbf{v}_{\varphi}, \mathbf{v}_{\theta}\right\rangle_{\perp \mathbf{e}}\right|^{2}}{\left\langle\mathbf{v}_{\theta}, \mathbf{v}_{\theta}\right\rangle_{\perp \mathbf{e}}\left\langle\mathbf{v}_{\varphi}, \mathbf{v}_{\varphi}\right\rangle_{\perp \mathbf{e}}} \quad \text { and } \quad r_{\xi}^{2}=\frac{\left|\tilde{\mathbf{e}}^{\dagger} \mathbf{v}_{\xi}\right|^{2}}{\left\|\mathbf{v}_{\xi}\right\|^{2}}
$$

the Cramer-Rao Bound for parameter $\xi$ adopts the simplified form

$$
C R B_{\xi, \xi}=\frac{1}{\gamma_{N}} \frac{1}{\left(1-\rho^{2}\right)} \frac{1}{\left\|\mathbf{v}_{\xi}\right\|^{2}\left(1-r_{\xi}^{2}\right)}
$$

As shown in Appendix A, one can see that the coupling correlation coefficient $\rho^{2}$ is common to both CRB, while the expression of $r_{\xi}^{2}$ depends upon the estimated angle. The interest of this formulation is twofold. First hand, it splits each 
scalar CRB into three distinct angle related components, easing further analysis of the array DOA estimation performances regarding its configuration. Secondly, these two metrics explicitly expose the numerical stability of both $C R B_{\xi, \xi}$ (always positive according to the Schwarz inequality).

The optimization criterion presented in next section consists in minimizing $\rho^{2}$ and $r_{\xi}^{2}$ in (6). It is closer to an iterative adaptation of the sensors positions for considered DOA areas to extract inherent geometric principles than a strictly speaking mathematical optimization. The aim is to highlight the role of $\rho^{2}$ and $r_{\xi}^{2}$ played in the CRB variations with respect to array configurations rather than to deal with the analytic formulations of these metrics.

\section{ARray CONFiguration ANalysis based on CRB METRICS}

In this section we first confirm both $C R B_{\theta, \theta}$ and $C R B_{\varphi, \varphi}$ expressions through MUSIC Monte-Carlo simulations, since this algorithm has been proved to be asymptotically efficient in the single source case [11]. We next address the problem of array configuration optimization regarding source DOA based on metrics defined in Section IV.

As seen in (6), scalar CRB both depend on SNR (through $\left.\gamma_{N}\right)$, array configuration parameters $(\boldsymbol{\Omega}, r)$ and DOA vector $\boldsymbol{\Theta}$. Hereafter simulations thus concern SNR and $\boldsymbol{\Omega}$ variations for nearly random values of $\Theta$. We deliberately indeed avoided DOA values that would raise trivial ambiguities due to array symmetry [12], not considered in this paper.

In what follows the sphere radius is assumed fixed at a quarter wavelength to ensure spatial sampling conditions for non-exotic values of $\boldsymbol{\Omega}$. Each Mean Square Error (MSE) value is obtained through a 400 point Monte-Carlo simulation of $T=100$ samples. Figure 2 shows the evolution of both MUSIC estimation of $\theta$ and $\varphi$ with respect to SNR compared to $C R B_{\xi, \xi}$ for DOA $\Theta=(0,60)$ and array configuration $\boldsymbol{\Omega}=(80,10)$. Accordingly we will assume for next simulations that the MUSIC estimator here adopts its asymptotic behaviour above a threshold SNR of approximately $10 \mathrm{~dB}$.

Achieving CRB validation, Figure 3 displays the MUSIC estimation MSE of DOA $\boldsymbol{\Theta}=(0,60)$ compared to $C R B_{\xi, \xi}$ with respect to $\beta$ and $\eta$ for a $15 \mathrm{~dB}$ SNR.

Trivial behaviours already stand out, as the dependency of an azimuthal estimation on the $O x y$ array aperture (related to $\beta$ ), or the drop of performances in any estimation of $\varphi$ while $M_{2}$ comes closer to the horizontal plane (i.e. for large values of $\eta$ ). Next part extends these observations through 3D plots and analysis of $\rho^{2}$ and $r_{\xi}^{2}$ variations with respect to sensors locations.

An attentive examination of the variations of $\rho^{2}$ with respect to array configurations $\boldsymbol{\Omega}$ shows that this metric is related to the orthogonality of the considered source DOA regarding the array plane. As seen in Figure 4, the nulls of $\left(1-\rho^{2}\right)$ are located on DOAs coplanar to $M_{1} M_{2} M_{3}$, while it approaches 1 for orthogonal values of $\Theta$.

On the other hand, coefficients $r_{\theta}^{2}$ and $r_{\varphi}^{2}$ are to be considered as cost metrics, binding the considered array configuration

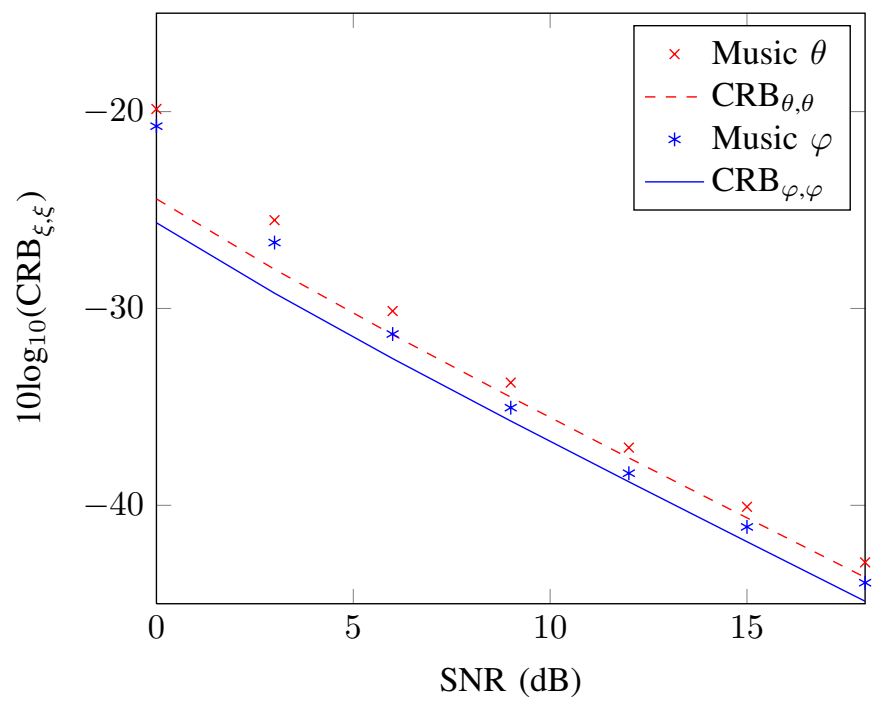

Fig. 2. Variations of $C R B_{\theta, \theta}, C R B_{\varphi, \varphi}$ and MSE of MUSIC DOA estimation of $\theta$ and $\varphi$ with respect to SNR for fixed array configuration $\boldsymbol{\Omega}=(80,10)$ and DOA $\boldsymbol{\Theta}=(0,60)$.

to its would have been configuration for a 2D DOA estimation. To be more specific, it is the whole term $\left\|\mathbf{v}_{\xi}\right\|^{2}\left(1-r_{\xi}^{2}\right)$ that has to be considered to pursue the analogy. These coefficients (for $\xi \in\{\theta, \varphi\}$ ) concern the DOA estimation efficiency once projected on the azimuthal or elevation planes. The analysis is simpler for $\xi=\theta$, since a projection on the azimuthal plane is very much alike one on the $O x y$ plane (while the elevational plane depends on $\theta$ ). Thus Figure 5 represents two spherical plots of $\left\|\mathbf{v}_{\theta}\right\|^{2}\left(1-r_{\theta}^{2}\right)$ for $\boldsymbol{\Omega}=(90,20)$ and $(10,20)$, showing that as $\beta$ drops the main lobe progressively shifts form $\theta=0$ to $\frac{\pi}{2}$. The shift occurs when the projection of $\left\{M_{1}, M_{2}, M_{3}\right\}$ in the azimuthal plane is an equilateral triangle, i.e. when $\mathbf{M}_{1} \mathbf{M}_{2} \cdot \mathbf{O x}=\left\|\mathbf{M}_{1} \mathbf{M}_{2}\right\| \cos \left(\frac{\pi}{6}\right)$, which would correspond to the isotropic condition for a $2 \mathrm{D}$ estimation on $\theta$. Observing each colour bar, one may also observe the awaited drop of $\left\|\mathbf{v}_{\theta}\right\|^{2}\left(1-r_{\theta}^{2}\right)$ maxima while the array characteristic length goes from half to a quarter wavelength.

The same analysis eventually holds for $\xi=\varphi$. Since the projection of $\left\{M_{1}, M_{2}, M_{3}\right\}$ on the elevational plane is less intuitive, Figure 6 mimics Figure 5 for the term $\left\|\mathbf{v}_{\varphi}\right\|^{2}(1-$ $\left.r_{\varphi}^{2}\right)$. As for $\theta$, the general orientation of the antenna array toward $O y z$ or $O z x$ defines the main lobe position, partially conditioning DOA estimation acuteness regarding $\varphi$.

These results are in accordance with those of Gazzah [8] and Dogandzic [13] , who observed that least and most desirable DOAs are perpendicular to each other and correspond to the antenna array "main" axes of symmetry (termed principal axes of inertia in [13]).

Finally, Figures 7 and 8 present both products $\left\|\mathbf{v}_{\xi}\right\|^{2}(1-$ $\left.r_{\xi}^{2}\right)\left(1-\rho^{2}\right)$, i.e. the terms of interest for each considered $C R B_{\xi, \xi}$ in (6) regarding angular dependency of the DOA estimation acuteness relative to sensors positions. Reflecting the CRBs behaviours for various array configurations, these 


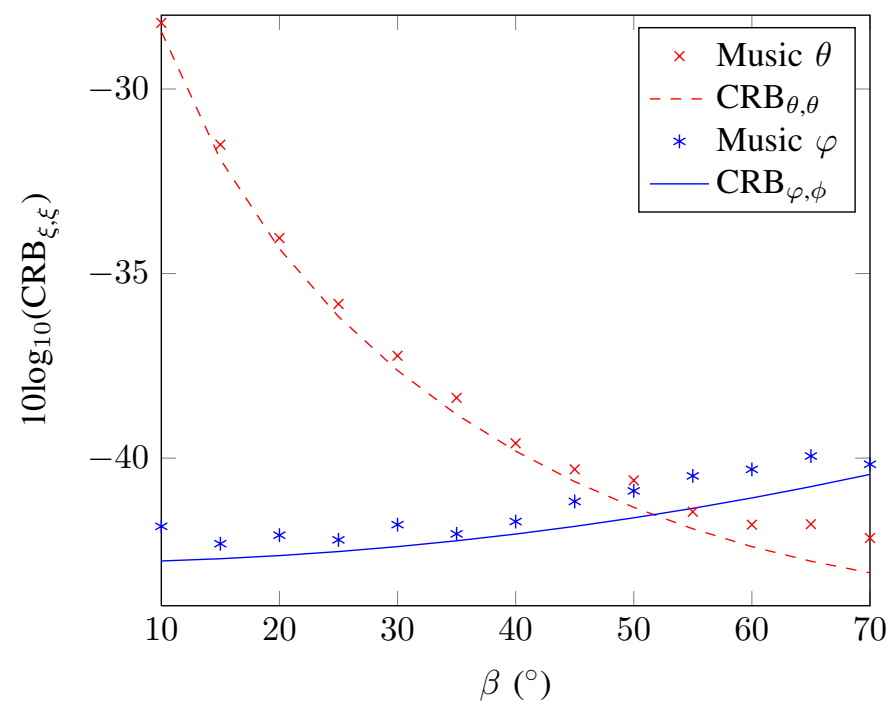

(a)

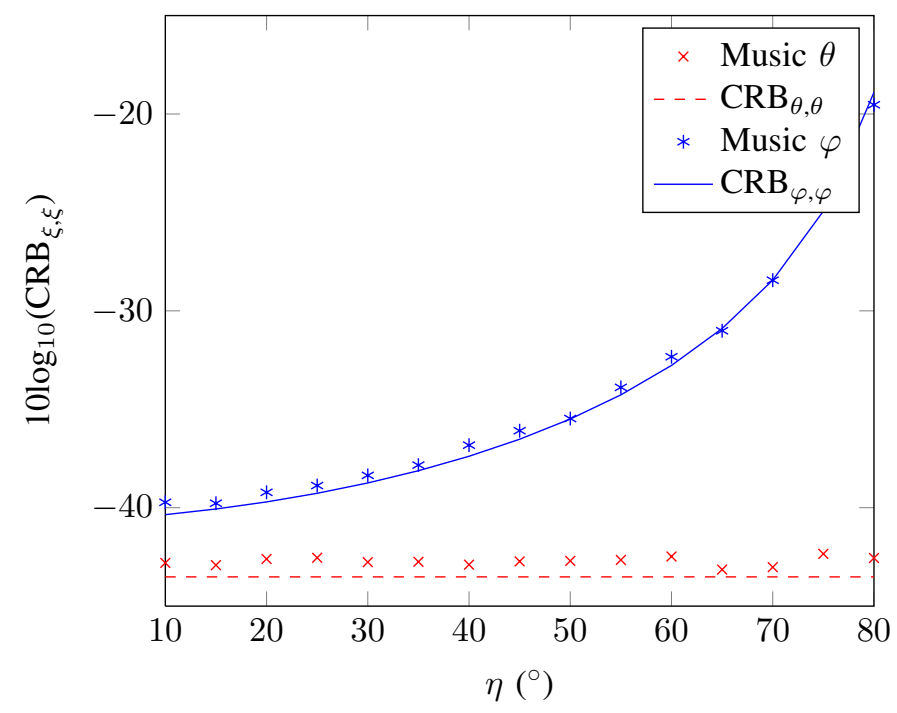

(b)

Fig. 3. Variations of $C R B_{\theta, \theta}, C R B_{\varphi, \varphi}$ and MSE of MUSIC estimation of $\theta$ and $\varphi$ regarding (a) $\beta$ and (b) $\eta$ for fixed $\eta=0^{\circ}$ and $\beta=60^{\circ}$ respectively. DOA is fixed at $\Theta=(0,60)$ for a $15 \mathrm{~dB}$ SNR.

representations are to be afterwards exploited in the real time design optimization process.

Based on these results, one may iteratively adapt the antenna array geometry to various research scenarios, assuming rough DOA and distance measurements feedback. From afar, the victim's elevation with respect to the man held antenna array approaches zero, while it may obviously be positioned anywhere in the horizontal plane. Preferred configurations thus would be those granting a low and constant $C R B_{\theta, \theta}$ for $\varphi=0$ and $\theta \in[0,2 \pi]$. While the array geometry constraints and its reduced number of elements prevent isotropic estimations on $\theta$, the above-mentioned equilateral condition would here represent the most favourable configuration, giving a pattern close to the one depicted in Figure 7(b). For building like environments, $\Theta$ is likely to assume values in the upper or

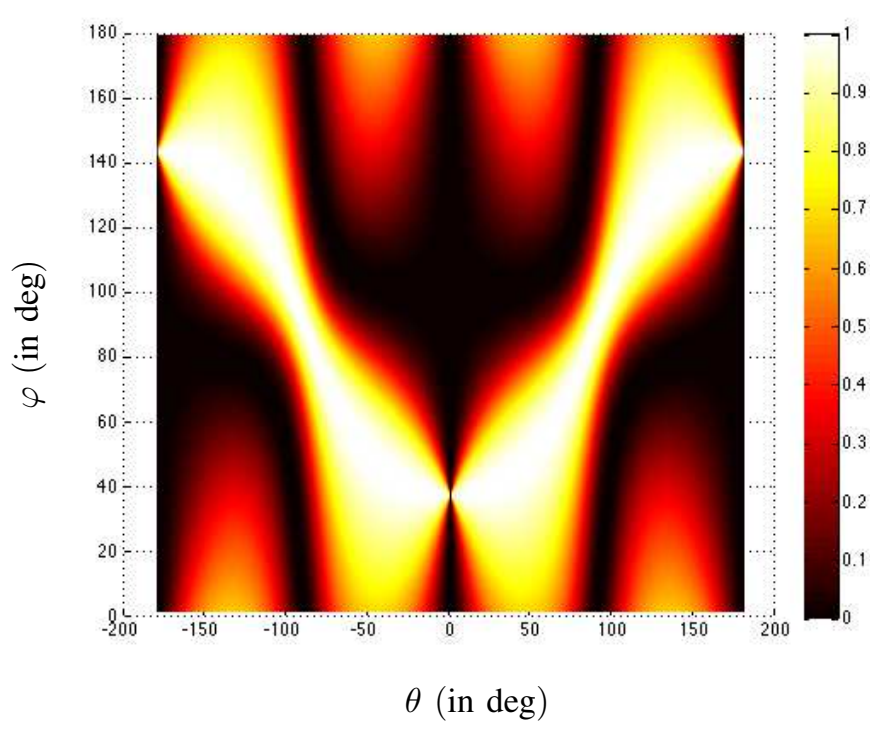

Fig. 4. Variations of $\rho^{2}$ along $\theta$ and $\varphi$ for $\boldsymbol{\Omega}=(80,45)$. The least desirable DOAs are for $\rho^{2}=1$, i.e for a source in the antenna array plane $M_{1} M_{2} M_{3}$.

lower front quadrants while the rescuer walks towards a victim potentially not on the same floor. $C R B_{\theta, \theta}$ main lobe (i.e. its minimum values) would thus have to be iteratively spread towards estimated DOAs ((Figures 7(b) or (a)) while minimizing $C R B_{\varphi, \varphi}$ values in the expected quadrant (((Figures $8(\mathrm{~b})$ or (a)).

\section{CONCLUSION}

In this paper, we adapted the stochastic Cramer-Rao Bound analytic expression to a helmet-mounted antenna array configuration for the single source DOA estimation case. We decomposed the obtained CRBs into metrics to give physical insight on the DOA estimation acuteness with respect to array configurations. Array elements positioning being reduced to a 2 Degree-of-Freedom problem, a few spherical plots along with proposed metrics alleviate the FIM based design optimization. Observing simple geometrical rules relative to these metrics, one may control each $C R B_{\xi, \xi}$ main lobe position while avoiding critical precision scenarios for a given set of DOAs. Further analysis based on 2D parametric representations of $\rho^{2}$ against $r_{\xi}^{2}$ with respect to $\boldsymbol{\Theta}$ for a given $\boldsymbol{\Omega}$ might facilitate zones of interests (i.e. areas where both $\rho^{2}$ and $r_{\xi}^{2}$ approach zero) characterization. Along with network logistic and human-machine interface considerations not discussed in this paper, the presented helmet-mounted antenna array and its optimization tool are supposed to enhance rescuers efficiency to reach natural disaster survivors. General expressions herein proposed hold for any spherical array configuration in the single source case, and would gain in being extended to any array geometry to pursue metrics behaviour analysis.

\section{ACKNOWLEDGMENTS}

The work described in this paper was carried out as part of an ANRT-CIFRE industrial thesis funded by EADS Astrium 


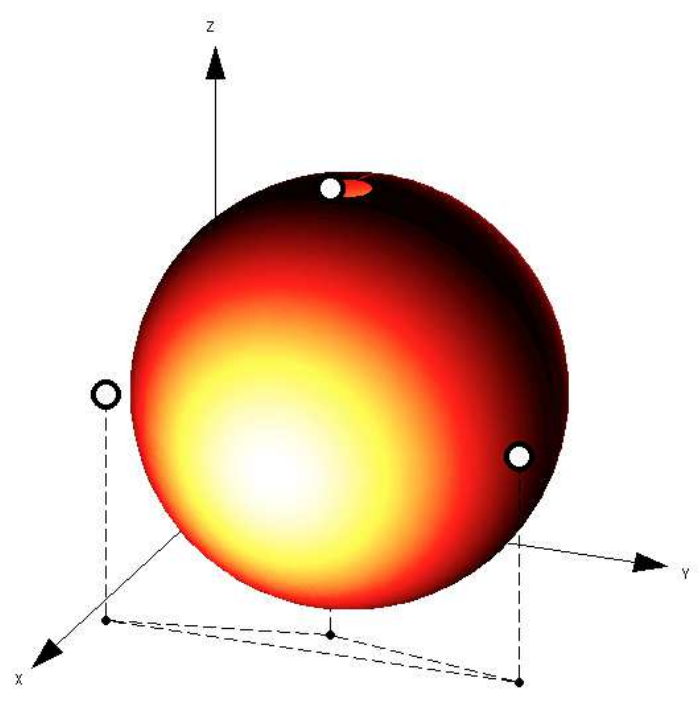

(a)

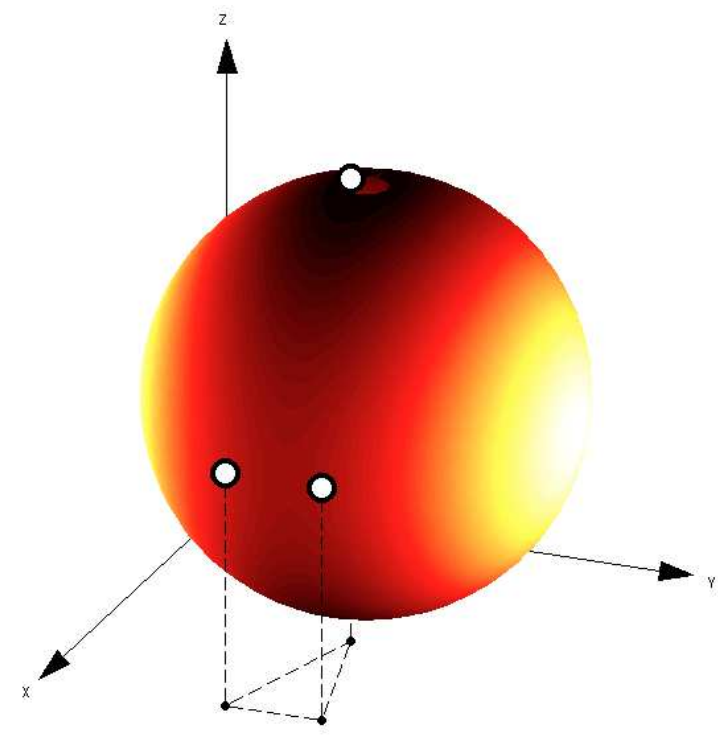

(b)
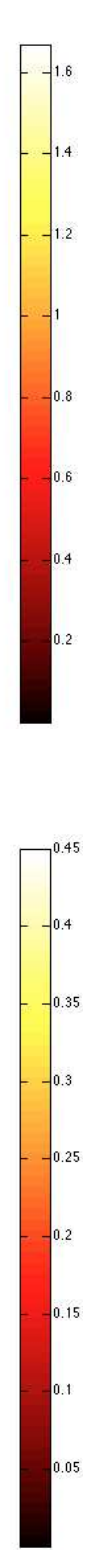

Fig. 5. Variations of $\left\|\mathbf{v}_{\theta}\right\|^{2}\left(1-r_{\theta}^{2}\right)$ on a sphere spanning $(\theta, \varphi)$ for (a) $\boldsymbol{\Omega}=(66,12)$ and (b) $(9,12)$. The main lobe inversion occurs when the azimuthal projections of $\left\{M_{1}, M_{2}, M_{3}\right\}$ (i.e. on $\left.O x y\right)$ form an equilateral triangle.

Services.

\section{APPENDIX}

This appendix holds an exhaustive demonstration of the FIM formulation of equation (3) used to establish $C R B_{\xi, \xi}$ scalar expressions in (6).

According to hypothesis stated in Section II, the probability density functions (pdf) of received snapshots $\mathbf{y}[t]$ along the $T$ measurements are independents. Their joint pdf thus takes the form

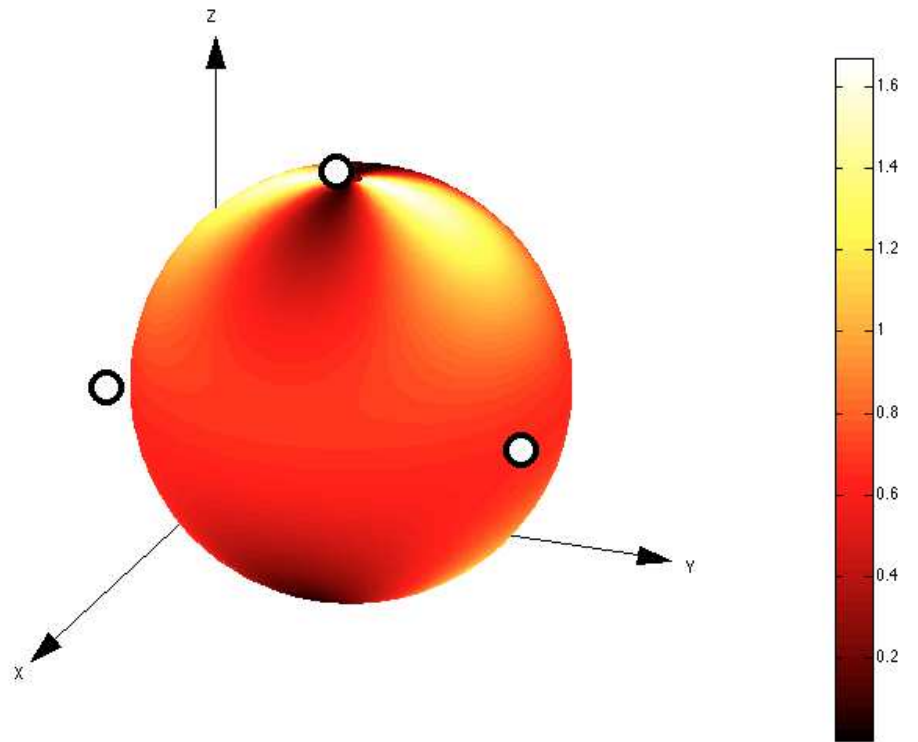

(a)

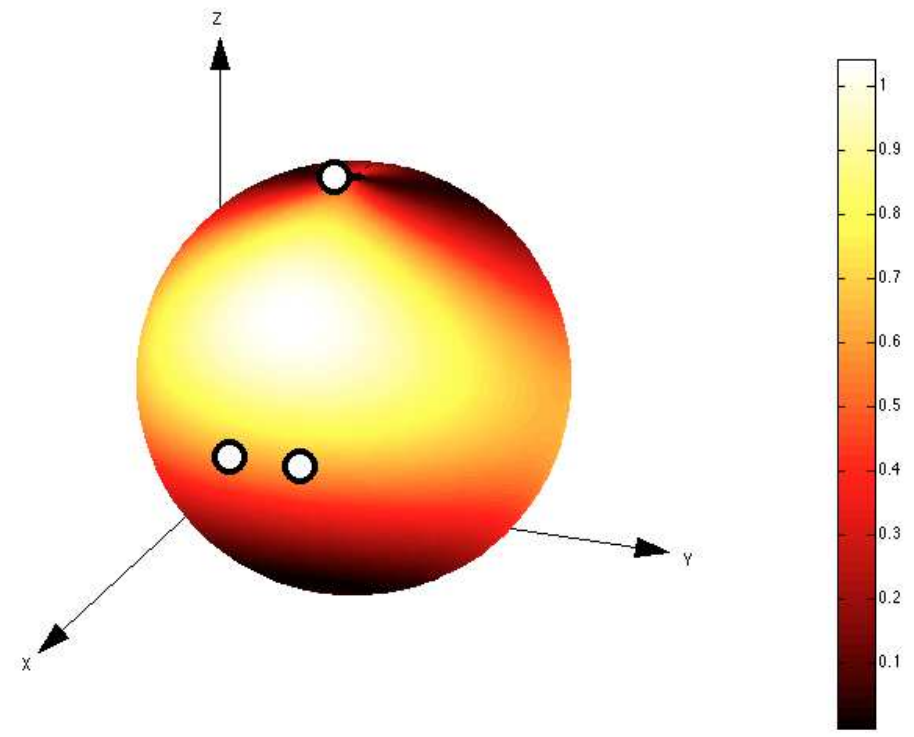

(b)

Fig. 6. Variations of $\left\|\mathbf{v}_{\varphi}\right\|^{2}\left(1-r_{\varphi}^{2}\right)$ on a sphere spanning $(\theta, \varphi)$ for (a) $\boldsymbol{\Omega}=(66,12)$ and (b) $(9,12)$. The main lobe inversion occurs when the elevational projections of $\left\{M_{1}, M_{2}, M_{3}\right\}$ form an equilateral triangle.

$\prod_{t=1}^{T} p\left(\mathbf{y}[t] \mid \Theta, \sigma_{s}^{2}, \sigma_{n}^{2}\right)=\frac{1}{\pi^{N T}} \frac{1}{\left|\boldsymbol{\Gamma}_{y}\right|^{T}} \exp \left(-\sum_{t=1}^{T} \mathbf{y}^{\dagger}[t] \boldsymbol{\Gamma}_{y}^{-1} \mathbf{y}[t]\right)$

Where the assumptions made on $s[t]$ and $\mathbf{n}[t]$ imply (using the steering vector simplified notation $\mathbf{e}=\mathbf{e}_{\boldsymbol{\Omega}}(\boldsymbol{\Theta})$ )

$$
\boldsymbol{\Gamma}_{y}=\sigma_{s}^{2} \mathbf{e e}^{\dagger}+\sigma_{n}^{2} \mathbf{I}_{N}
$$

$\boldsymbol{\Gamma}_{y}$ being the spatial covariance matrix of vector $\mathbf{y}$. The exponential term in (7) being a scalar, we may use the circular 


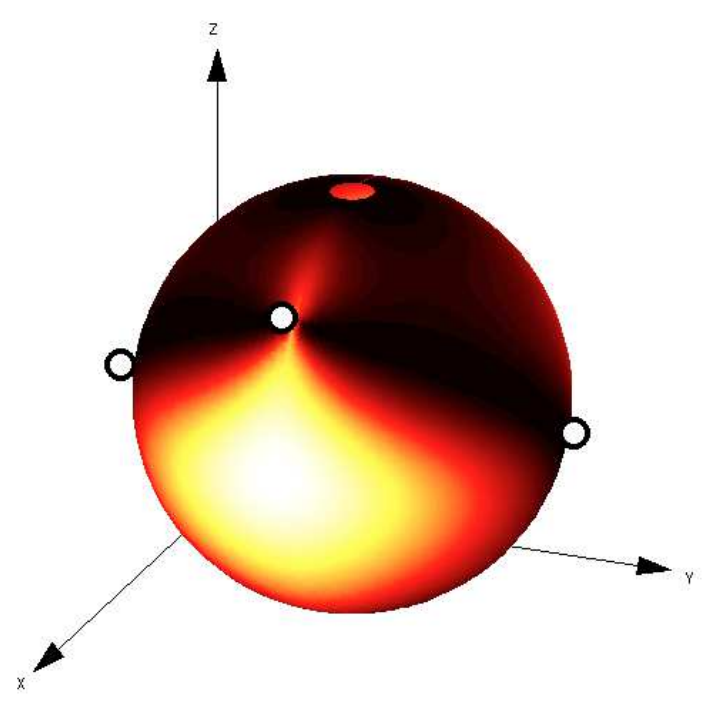

(a)

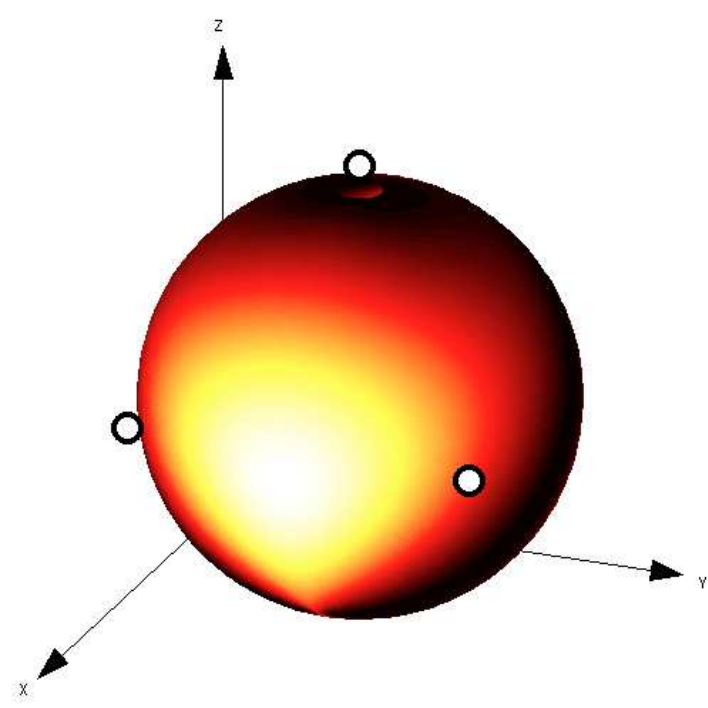

(b)

Fig. 7. Variations of $\left(1-\rho^{2}\right)\left\|\mathbf{v}_{\theta}\right\|^{2}\left(1-r_{\theta}^{2}\right)$ for (a) $\boldsymbol{\Omega}=(87,51)$ and (b) $(45,0)$. The general array orientation favors $\theta$ estimations on larger areas for DOAs orthogonal to the array plane.

permutation invariance of the trace operator to reveal the covariance estimation matrix $\hat{\boldsymbol{\Gamma}}_{y}$

$$
\begin{aligned}
\sum_{t=1}^{T} \mathbf{y}^{\dagger}[t] \boldsymbol{\Gamma}_{y}^{-1} \mathbf{y}[t] & =\operatorname{tr}\left(\sum_{t=1}^{T} \mathbf{y}[t] \mathbf{y}^{\dagger}[t] \boldsymbol{\Gamma}_{y}^{-1}\right) \\
& =\operatorname{tr}\left(T \hat{\boldsymbol{\Gamma}}_{y} \boldsymbol{\Gamma}_{y}^{-1}\right)
\end{aligned}
$$

where

$$
\hat{\boldsymbol{\Gamma}}_{y}=\frac{1}{T} \sum_{t=1}^{T} \mathbf{y}[t] \mathbf{y}[t]^{\dagger} \quad \text { with } \quad E\left[\hat{\boldsymbol{\Gamma}}_{y}\right]=\boldsymbol{\Gamma}_{y}
$$

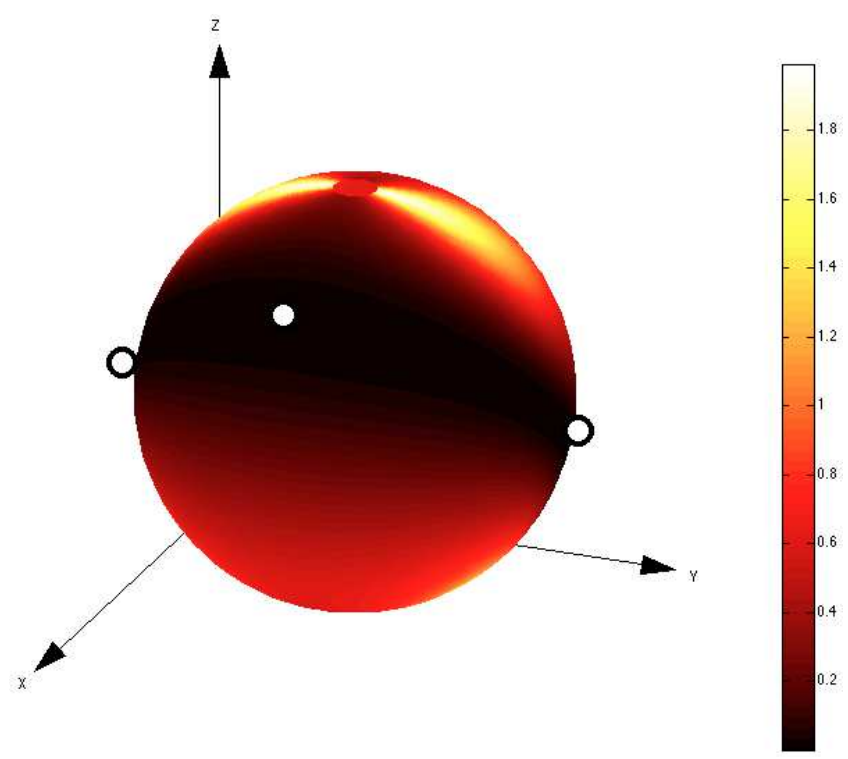

(a)

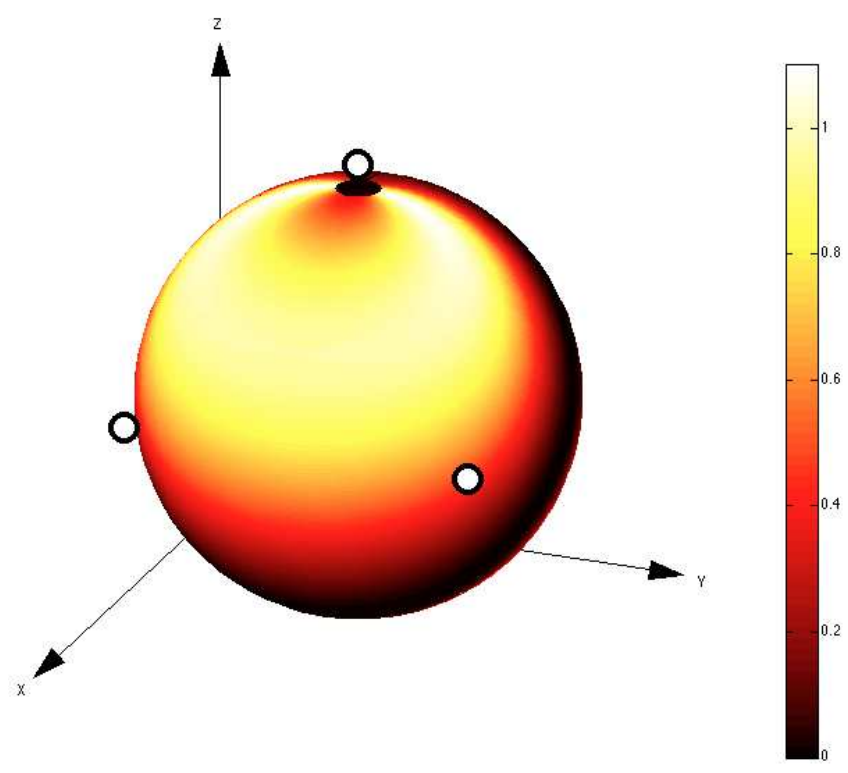

(b)

Fig. 8. Variations of $\left(1-\rho^{2}\right)\left\|\mathbf{v}_{\varphi}\right\|^{2}\left(1-r_{\varphi}^{2}\right)$ for (a) $\boldsymbol{\Omega}=(87,51)$ and (b) $(45,0)$. The general array orientation favors $\varphi$ estimations on larger areas for DOAs orthogonal to the array plane.

The basic formulation of the log-likelihood function is then obtained by replacing (9) in (7) and taking its natural logarithm

$$
\Lambda=-N T \log (\pi)-T \log \left|\boldsymbol{\Gamma}_{y}\right|-T \operatorname{tr}\left(\boldsymbol{\Gamma}_{y}^{-1} \hat{\boldsymbol{\Gamma}}_{y}\right)
$$

$\Lambda$ thus needs to be shaped for derivation regarding $\Theta$. Using (8) to replace $\left|\boldsymbol{\Gamma}_{y}\right|$

$$
\begin{aligned}
\left|\boldsymbol{\Gamma}_{y}\right|=\left|\sigma_{s}^{2} \mathbf{e e}^{\dagger}+\sigma_{n}^{2} \mathbf{I}_{N}\right| & =\sigma_{n}^{2(N-1)}\left(\sigma_{s}^{2} \mathbf{e e}^{\dagger}+\sigma_{n}^{2}\right) \\
& =\sigma_{n}^{2 N}(\alpha N+1)
\end{aligned}
$$

and $\Gamma_{y}^{-1}$ (according to Woodbury inversion formula) 


$$
\begin{aligned}
\operatorname{tr}\left(\boldsymbol{\Gamma}_{y}^{-1} \hat{\boldsymbol{\Gamma}}_{y}\right) & =\frac{1}{\sigma_{n}^{2}} \operatorname{tr}\left(\left[\alpha \mathbf{e e}^{\dagger}+\mathbf{I}_{N}\right]^{-1} \hat{\boldsymbol{\Gamma}}_{y}\right) \\
& =\frac{1}{\sigma_{n}^{2}} \operatorname{tr}\left(\left[\mathbf{I}_{N}-\frac{\alpha \mathbf{e} \mathbf{e}^{\dagger}}{1+\alpha N}\right] \hat{\boldsymbol{\Gamma}}_{y}\right)
\end{aligned}
$$

in (10), the log-likelihood becomes

$$
\begin{array}{r}
\Lambda=-N T\left(\log (\pi)+\log \left(\sigma_{n}^{2}\right)\right)-\frac{T}{\sigma_{n}^{2}} \operatorname{tr}\left(\hat{\boldsymbol{\Gamma}}_{y}\right) \\
-T \log (1+\alpha N)+\frac{T}{\sigma_{n}^{2}} \frac{\alpha}{(1+\alpha N)} \mathbf{e}^{\dagger} \hat{\boldsymbol{\Gamma}}_{y} \mathbf{e}
\end{array}
$$

Since the considered derivation concerns $\Theta$ dependent elements, only the last term of (11) is of interest. For the sake of clarity, derivatives like $\delta_{\xi}(\mathbf{e})$ or $\delta_{\xi, \zeta}^{2}\left(\mathbf{e}^{\dagger}\right)$ will thereafter be denoted as $\delta_{\xi} \mathbf{e}$ and $\delta_{\xi, \zeta}^{2} \mathbf{e}^{\dagger}$, the derivative affecting its adjacent term only. The first derivative of (11) with respect to $\xi \in\{\theta, \varphi\}$ is then

$$
\delta_{\xi} \Lambda=\frac{T}{\sigma_{n}^{2}} \frac{\alpha}{(1+\alpha N)}\left(\delta_{\xi} \mathbf{e}^{\dagger} \hat{\boldsymbol{\Gamma}}_{y} \mathbf{e}+\mathbf{e}^{\dagger} \hat{\boldsymbol{\Gamma}}_{y} \delta_{\xi} \mathbf{e}\right)
$$

the second derivative related to $\zeta \in\{\theta, \varphi\}$ naturally reveals cross conjugated terms, hereafter gathered in $\Re\{\cdot\}$

$$
\delta_{\xi, \zeta}^{2} \Lambda=2 \frac{T}{\sigma_{n}^{2}} \frac{\alpha}{(1+\alpha N)}\left(\Re\left\{\delta_{\xi, \zeta}^{2} \mathbf{e}^{\dagger} \hat{\boldsymbol{\Gamma}}_{y} \mathbf{e}\right\}+\Re\left\{\delta_{\xi} \mathbf{e}^{\dagger} \hat{\boldsymbol{\Gamma}}_{y} \delta_{\zeta} \mathbf{e}\right\}\right)
$$

According to the FIM definition stated in Section III, we need to evaluate the negative expectation of the second order derivative log-likelihood. Replacing the estimate $\hat{\boldsymbol{\Gamma}}_{y}$ by its true value $\boldsymbol{\Gamma}_{y}$ in (12) gives

$E\left[-\delta_{\xi, \zeta}^{2} \Lambda\right]=-2 \frac{T}{\sigma_{n}^{2}} \frac{\alpha}{(1+\alpha N)}\left(\Re\left\{\delta_{\xi, \zeta}^{2} \mathbf{e}^{\dagger} \boldsymbol{\Gamma}_{y} \mathbf{e}\right\}+\Re\left\{\delta_{\xi} \mathbf{e}^{\dagger} \boldsymbol{\Gamma}_{y} \delta_{\zeta} \mathbf{e}\right\}\right)$

which once developed according to (8) gives

$$
\begin{aligned}
E\left[-\delta_{\xi, \zeta}^{2} \Lambda\right]= & -\frac{2 \alpha T}{(1+\alpha N)}\left[(1+\alpha N) \Re\left\{\delta_{\xi, \zeta}^{2} \mathbf{e}^{\dagger} \mathbf{e}\right\}\right. \\
& \left.+\Re\left\{\delta_{\xi} \mathbf{e}^{\dagger} \delta_{\zeta} \mathbf{e}\right\}+\alpha \Re\left\{\delta_{\xi} \mathbf{e}^{\dagger} \mathbf{e e}^{\dagger} \delta_{\zeta} \mathbf{e}\right\}\right]
\end{aligned}
$$

We then develop the differential terms $\delta_{\xi} \mathbf{e}^{\dagger}$ and $\delta_{\xi, \zeta}^{2} \mathbf{e}^{\dagger}$, introducing the notations

$$
\mathbf{e}=\left(\begin{array}{c}
e^{j \frac{2 \pi r}{\lambda} u_{1}} \\
e^{j \frac{2 \pi r}{\lambda} u_{2}} \\
\vdots \\
e^{j \frac{2 \pi r}{\lambda} u_{N}}
\end{array}\right) \text { and } \mathbf{U}=\left(\begin{array}{cccc}
u_{1} & 0 & \ldots & 0 \\
0 & u_{2} & \ldots & 0 \\
\vdots & \vdots & \ddots & \vdots \\
0 & 0 & \ldots & u_{N}
\end{array}\right)
$$

we get the first and second order derivatives

$$
\begin{aligned}
& \delta_{\xi} \mathbf{e}^{\dagger}=-j \frac{2 \pi r}{\lambda} \mathbf{e}^{\dagger} \delta_{\xi} \mathbf{U} \\
& \delta_{\xi, \zeta}^{2} \mathbf{e}^{\dagger}=-j \frac{2 \pi r}{\lambda} \mathbf{e}^{\dagger} \delta_{\xi, \zeta}^{2} \mathbf{U}-\left(\frac{2 \pi r}{\lambda}\right)^{2} \mathbf{e}^{\dagger} \delta_{\xi} \mathbf{U} \delta_{\zeta} \mathbf{U}
\end{aligned}
$$

used to develop (13) into

$$
-E\left[\delta_{\xi, \zeta}^{2} \Lambda\right]=\frac{\gamma_{N}}{N}\left(\mathbf{e}^{\dagger} \mathbf{e} \mathbf{e}^{\dagger} \delta_{\zeta} \mathbf{U} \delta_{\xi} \mathbf{U} \mathbf{e}-\mathbf{e}^{\dagger} \delta_{\zeta} \mathbf{U e e}^{\dagger} \delta_{\xi} \mathbf{U e}\right)
$$

with

$$
\gamma_{N}=2 \alpha T \frac{1}{\left(1+\frac{1}{\alpha N}\right)}\left(\frac{2 \pi r}{\lambda}\right)^{2}
$$

Remark here that $E\left[\delta_{\xi, \zeta}^{2} \Lambda\right]=E\left[\delta_{\zeta, \xi}^{2} \Lambda\right]$, since $\mathbf{U}$ is diagonal with real coefficients and $\mathbf{e e}^{\dagger}$ is a hermitian matrix. Noticing the weighted scalar product in (14)

$$
\begin{aligned}
\left(\mathbf{e}^{\dagger} \mathbf{e} \mathbf{e}^{\dagger} \delta_{\zeta} \mathbf{U} \delta_{\xi} \mathbf{U e}-\mathbf{e}^{\dagger} \delta_{\zeta} \mathbf{U} \mathbf{e} \mathbf{e}^{\dagger} \delta_{\xi} \mathbf{U e}\right) & =\mathbf{e}^{\dagger} \mathbf{e}\left(\mathbf{v}_{\zeta}^{\dagger} \boldsymbol{\Pi}_{\perp \mathbf{e}} \mathbf{v}_{\xi}\right) \\
& =N\left\langle\mathbf{v}_{\xi}, \mathbf{v}_{\zeta}\right\rangle_{\perp} \mathbf{e}
\end{aligned}
$$

where $\boldsymbol{\Pi}_{\perp \mathbf{e}}$ is the orthonormal projector on the noise space defined in the MUSIC subspace decomposition

$$
\Pi_{\perp \mathbf{e}}=\mathbf{I}_{\mathbf{N}}-\tilde{\mathbf{e}} \tilde{\mathbf{e}}^{\dagger} \quad \text { with } \quad \tilde{\mathbf{e}}=\frac{\mathbf{e}}{\sqrt{\mathbf{e}^{\dagger} \mathbf{e}}}
$$

equation (14) becomes

$$
-E\left[\delta_{\xi, \zeta}^{2} \Lambda\right]=\gamma_{N}\left\langle\mathbf{v}_{\xi}, \mathbf{v}_{\zeta}\right\rangle_{\perp \mathbf{e}}
$$

which proves the proposed FIM in (3):

$$
F(\boldsymbol{\Theta})=\gamma_{N}\left(\begin{array}{c}
\left\langle\mathbf{v}_{\theta}, \mathbf{v}_{\theta}\right\rangle_{\perp \mathbf{e}}\left\langle\mathbf{v}_{\theta}, \mathbf{v}_{\varphi}\right\rangle_{\perp \mathbf{e}} \\
\left\langle\mathbf{v}_{\varphi}, \mathbf{v}_{\theta}\right\rangle_{\perp \mathbf{e}}\left\langle\mathbf{v}_{\varphi}, \mathbf{v}_{\varphi}\right\rangle_{\perp \mathbf{e}}
\end{array}\right)
$$

or its equivalent vectorial form that highlights the MUSIC noise space projection

$$
F(\Theta)=\gamma_{N}\left(\begin{array}{c}
\mathbf{v}_{\theta}^{\dagger} \\
\mathbf{v}_{\varphi}^{\dagger}
\end{array}\right) \Pi_{\perp \mathbf{e}}\left(\mathbf{v}_{\theta} \mathbf{v}_{\varphi}\right)
$$

Afterward, a simple inversion of (3) reveals the determinant $\left(1-\rho^{2}\right)=\left\langle\mathbf{v}_{\theta}, \mathbf{v}_{\theta}\right\rangle_{\perp \mathbf{e}} \cdot\left\langle\mathbf{v}_{\varphi}, \mathbf{v}_{\varphi}\right\rangle_{\perp \mathbf{e}}-\left\langle\mathbf{v}_{\theta}, \mathbf{v}_{\varphi}\right\rangle_{\perp \mathbf{e}} \cdot\left\langle\mathbf{v}_{\varphi}, \mathbf{v}_{\theta}\right\rangle_{\perp \mathbf{e}}$ and leads to the CRB matrix, which holds both $C R B_{\xi, \xi}$ diagonal terms introduced in (6)

$$
\begin{aligned}
& F(\boldsymbol{\Theta})^{-1}=\frac{1}{\gamma_{N}} \frac{1}{\left(1-\rho^{2}\right)}\left(\begin{array}{cc}
\left\langle\mathbf{v}_{\theta}, \mathbf{v}_{\theta}\right\rangle_{\perp \mathbf{e}}^{-1} & -\rho^{2}\left\langle\mathbf{v}_{\theta}, \mathbf{v}_{\varphi}\right\rangle_{\perp}^{-1} \\
-\rho^{2}\left\langle\mathbf{v}_{\varphi}, \mathbf{v}_{\theta}\right\rangle_{\perp \mathbf{e}}^{-1}\left\langle\mathbf{v}_{\varphi}, \mathbf{v}_{\varphi}\right\rangle_{\perp \mathbf{e}}^{-}
\end{array}\right) \\
& \quad \text { where }\left\langle\mathbf{v}_{\xi}, \mathbf{v}_{\xi}\right\rangle_{\perp \mathbf{e}}=\mathbf{v}_{\xi}^{\dagger}\left(\mathbf{I}_{\mathbf{N}}-\tilde{e} \tilde{e}^{\dagger}\right) \mathbf{v}_{\xi}=\left\|\mathbf{v}_{\xi}\right\|\left(1-r_{\xi}^{2}\right) \text { for } \\
& \xi \in\{\theta, \varphi\} .
\end{aligned}
$$




\section{REFERENCES}

[1] C. Fischer and H. Gellersen, "Location and navigation support for emergency responders: A survey," IEEE Pervasive Computing, vol. 9, pp. 38-47, 2010.

[2] R. Schmidt, "Multiple emitter location and signal parameter estimation," Antennas and Propagation, IEEE Transactions on, vol. 34, pp. 276 280, mar 1986.

[3] R. Roy and T. Kailath, "Esprit estimation of signal parameters via rotational invariance techniques," Acoustics, Speech and Signal Processing, IEEE Transactions on, vol. 37, pp. 984 -995, jul 1989.

[4] M. Viberg and U. i Linkping. Dept. of Electrical Engineering, Subspace Fitting Concepts in Sensor Array Processing. Linkping studies in science and technology: Disertations, Department of Electrical Engineering, Linkping University, 1989.

[5] A. Manikas, A. Alexiou, and H. Karimi, "Comparison of the ultimate direction-finding capabilities of a number of planar array geometries," Radar, Sonar and Navigation, IEE Proceedings -, vol. 144, pp. 321 329, dec 1997.

[6] J. Verhaevert, E. Van Lil, and A. Van de Capelle, "Uniform spherical distributions for adaptive array applications," in Vehicular Technology Conference, 2001. VTC 2001 Spring. IEEE VTS 53rd, vol. 1, pp. 98 -102 vol.1, 2001

[7] A. Ferréol and P. Chevalier, "High resolution direction finding: From performance toward antenna array optimization- the mono-source case," EUSIPCO, 2009.

[8] H. Gazzah and S. Marcos, "Cramer-rao bounds for antenna array design," Signal Processing, IEEE Transactions on, vol. 54, pp. $336-$ 345, jan. 2006.

[9] P. Stoica, E. Larsson, and A. Gershman, "The stochastic crb for array processing: a textbook derivation," Signal Processing Letters, IEEE, vol. 8, pp. $148-150$, may 2001.

[10] R. A. Fisher, "The logic of inductive inference," Journal of the Royal Statistical Society, vol. 98, no. 1, pp. 39-82, 1935.

[11] B. Porat and B. Friedlander, "Analysis of the asymptotic relative efficiency of the music algorithm," in IEEE Trans. Acoustics, Speech, and Signal Processing, vol. ASSP-36, pp. 532-544, April 1988.

[12] C. Proukakis and A. Manikas, "Study of ambiguities of linear arrays," in Acoustics, Speech, and Signal Processing, 1994. ICASSP-94., 1994 IEEE International Conference on, vol. iv, pp. IV/549 -IV/552 vol.4, apr 1994.

[13] A. Dogandzic and A. Nehorai, "Cramer-rao bounds for estimating range, velocity, and direction with an active array," IEEE Transactions on Signal Processing, vol. 49, pp. 1122 -1137, June 2001. 